\title{
BMJ Open The effect of chronic, non-specific low back pain on superficial lumbar muscle activity: a protocol for a systematic review and meta-analysis
}

\author{
Andy Sanderson (D) , Alison B Rushton (D), Eduardo Martinez Valdes, \\ Nicola R Heneghan (D) , Alessio Gallina, Deborah Falla (D)
}

To cite: Sanderson A, Rushton AB, Martinez Valdes $E$ et al. The effect of chronic, non-specific low back pain on superficial lumbar muscle activity: a protocol for a systematic review and meta-analysis. BMJ Open 2019;9:e029850. doi:10.1136/ bmjopen-2019-029850

- Prepublication history and additional material for this paper are available online. To view these files, please visit the journal online (http://dx.doi org/10.1136/bmjopen-2019029850).

Received 14 February 2019 Revised 09 September 2019 Accepted 15 October 2019

A Check for updates

(c) Author(s) (or their employer(s)) 2019. Re-use permitted under CC BY-NC. No commercial re-use. See rights and permissions. Published by BMJ.

Centre of Precision

Rehabilitation for Spinal Pain (CPR Spine), School of Sport, Exercise and Rehabilitation Sciences, College of Life and Environmental Sciences, University of Birmingham, Birmingham, UK

Correspondence to Professor Deborah Falla; d.falla@bham.ac.uk

\section{ABSTRACT}

Introduction Chronic, non-specific low back pain is a major global cause of disability. One factor which might potentially contribute to ongoing pain is maladaptive variation in the level of activity in the lumbar musculature. Several studies have investigated this activity using surface electromyography, in varied muscles and during a number of functional activities. Due to differences in the applied methodology, the results have been difficult to compare, and previous reviews have been limited in scope. In this protocol, we aim to perform a comprehensive review of the effect of chronic low back pain on lumbar muscle activity.

Methods and analysis This protocol was informed by the Preferred Reporting Items for Systematic Review and Meta-analysis Protocols (PRISMA-P) and results will be reported in line with the PRISMA. Searches will be conducted on the Web of Science, PubMed, MEDLINE, EMBASE, ZETOC and CINAHL databases, along with a comprehensive review of grey literature and key journals. One reviewer will conduct the searches, but two independent reviewers will screen potential studies and assess the risk of bias within studies which meet the inclusion criteria. The Newcastle-0ttawa risk of bias tool, and the Grading of Recommendations, Assessment, Development and Evaluation (GRADE) guidelines will be used to assess the quality of the data. Meta-analysis will be conducted where appropriate on groups of studies with homogenous methodology. Where studies are too heterogeneous to allow for meta-analysis, meta-synthesis will instead be completed, comparing results in terms of net increases or decreases of activity.

Ethics and dissemination This review aims to identify common adaptations of muscle activity in people with low back pain and it is expected that the results will influence future research directions and future rehabilitation approaches. The results will be submitted for publication in a peer-reviewed journal and presented at conferences. Prospero registration number CRD42019125156

\section{INTRODUCTION}

\section{Rationale}

Chronic low back pain (LBP) is a leading cause of disability, placing a high burden on healthcare services globally. ${ }^{12}$ Recent point
Strengths and limitations of this study

- First wide systematic search considering the effect of chronic, non-specific low back pain on muscle activity.

- This methodology allows for a wide range of tasks to be considered and included in meta-analysis.

- This protocol allowed for peer-review of the review methodology and so increases transparency and reduces bias.

- This protocol only accounts for observational studies, excluding randomised controlled trials, so the expected evidence level will be lower.

- While it would be beneficial to also consider the results of intramuscular electromyography, as the outcomes are too different, these results will be omitted.

prevalence estimates suggest that at any one time, approximately 540 million people are experiencing 'activity-limiting' LBP. ${ }^{3}$ Previous evidence suggests that of all those who experience LBP, $30 \%-40 \%$ will continue to experience symptoms beyond 3 months and thus become chronic. ${ }^{45}$ Furthermore, as many as $85 \%$ of those who experience any form of LBP will have no diagnosable underlying pathology and are thus categorised as experiencing 'non-specific' LBP. ${ }^{6}$ Therefore, efforts in healthcare research have included a focus on understanding the mechanisms underlying the maintenance of pain in chronic non-specific LBP (CNSLBP).

One potential physical contributing factor to LBP is variation in the level of activity of the lumbar paraspinal extensor muscles. This has the potential to change the load distribution on spinal structures, and thus contribute to the maintenance and/or perpetuation of pain. $^{7}$ However, despite several investigations, the exact link between the level of muscle activity and CNSLBP 
remains unclear. $^{7-10}$ The lumbar region is highly muscular, comprising subcutaneous contributions from the lumbar erector spinae (iliocostalis, longissimus and spinalis) and the superficial multifidus. ${ }^{11}$ These muscles are considered particularly important due to their role in nearly all functional spinal movements and postural control tasks. ${ }^{12} 13$ The most common technique used to investigate lumbar muscle activity is electromyography (EMG). ${ }^{14-16}$

EMG enables an understanding of muscles by recording the electrical activity during muscle contractions. ${ }^{17}$ This activity can be measured using skin mounted electrodes, termed surface EMG (sEMG), or via needle electrodes known as intramuscular EMG. Surface EMG offers a noninvasive means to record the level of activity from the superficial musculature, either between two points on a muscle (bipolar sEMG) or across a 2D region of the muscle (high-density sEMG; HDEMG). ${ }^{17}$ As sEMG is less invasive, it is more clinically useful and so studies which have investigated the effect of low back pain on muscle activity have often used sEMG electrodes over intramuscular techniques. ${ }^{18}$ However, direct comparison between results remains challenging as these studies have incorporated varied methodologies and consequently often reported varied results including both increases and decreases in muscle activity as a result of LBP. These studies have used bipolar and HDEMG electrodes to measure activity within differing lumbar musculature as participants completed a variety of tasks including fatiguing, walking and endurance contractions. ${ }^{14} 1519-22$

Previous reviews have attempted to synthesise these results to draw conclusions; however, these reviews have been focused on the investigation of activity within just one muscle, or during one task, for example, standing on unstable surfaces, or synthesising results drawn from one database. $^{8102324}$ While these reviews do answer specific questions, there remains a gap in the literature to assess what the overall effect of CNSLBP is on lumbar paraspinal muscle activity. In the most recent review of multiple tasks on muscle activity, with studies drawn from just one database, it was noted that differences were apparent in muscle activation between sitting and standing tasks in individuals with LBP. ${ }^{8}$ Despite this finding, no previous review has utilised a systematic search strategy to compile these results across tasks and understand the gross effect of CNSLBP on lumbar muscle activity.

Thus, there remains no comprehensive systematic review to date which assesses the effect of CNSLBP on the level of muscle activity across all superficial lumbar musculature during a variety of functional tasks. Understanding the differences in muscle activity between individuals with CNSLBP and pain-free controls is relevant since understanding the most common adaptation of lumbar muscle activity to pain may lead to the development of new rehabilitation approaches for use in clinical practice. $^{18}$

\section{Objectives}

1. To explore what differences are present in the activity of the superficial lumbar paraspinal musculature during functional tasks in individuals with CNSLBP, as measured by surface EMG.

If possible, dependant on the results of the primary objective and specifically if the data are suitably homogenous to allow meta-analysis, a secondary objective will be investigated.

2. To quantify the magnitude of changes in muscle activity associated with CNSLBP with regard to the type of functional task completed and the intensity/duration of LBP symptoms.

\section{METHODS AND ANALYSIS}

The protocol for this review has been developed in accordance with the Cochrane Back Review Group guidelines, the Cochrane Handbook, and the PRISMA-P preferred reporting guidelines (see online supplementary file 1). ${ }^{25-28}$ This protocol has been registered on PROSPERO (CRD42019125156) on the 12/02/2019.

\section{Eligibility criteria}

The PICOS framework has been used to inform the eligibility criteria for the inclusion and exclusion of studies. ${ }^{2829}$

\section{Population}

The population of interest is adults (aged 18 years or older) with chronic, non-specific (with no diagnosable underlying pathology) LBP, or adults who are pain-free and included in the context of a comparative control. For the purpose of this review, the definition for chronicity in LBP will follow the minimum guidelines by Dionne $e t a l$, and include pain in the lower back which has persisted for more than 3 months. ${ }^{30}$

\section{Intervention/Exposure}

The intervention of interest is the use of surface EMG to measure the level of activity of the lumbar musculature. This will include any measure of activity in any lumbar paraspinal muscle which can be (or has been) measured by surface EMG, including, but not limited to, the lumbar erector spinae or any of its component parts and the lumbar multifidus. ${ }^{831} 32$

\section{Comparison}

Studies must include a comparison of muscle activity related to a functional task. This comparison could be within-groups or between-groups and could include pre-task and post-task measures; continuous or multiple measurements throughout a task; or associated with a change in symptoms. ${ }^{33}$

\section{Outcomes}

The outcome of interest is the measurement of the amplitude of lumbar paraspinal muscle activity. Any measure related to the amplitude of muscle activity will be included, including root mean square (RMS), average rectified 
Table 1 Summary of Inclusion and exclusion criteria

\begin{tabular}{|c|c|}
\hline \multicolumn{2}{|l|}{ Inclusion criteria } \\
\hline Population & Adults (>18y), men and women with CNSLBP or a pain-free control \\
\hline Intervention/Exposure & $\begin{array}{l}\text { Use of surface EMG to measure the amplitude of activity of the lumbar paraspinal } \\
\text { musculature }\end{array}$ \\
\hline Comparison & $\begin{array}{l}\text { Differences in the magnitude of lumbar muscle activity between individuals with CNSLBP } \\
\text { and pain-free controls }\end{array}$ \\
\hline Outcomes & Surface EMG measurement of the amplitude of muscle activity \\
\hline \multicolumn{2}{|l|}{ Exclusion Criteria } \\
\hline Population & $\begin{array}{l}\text { Studies where individuals under the age of } 18 \text { were explicitly included } \\
\text { Individuals with LBP defined by study authors as 'Chronic' but has not persisted for over } \\
3 \text { months } \\
\text { Individuals with CNSLBP who have been diagnosed with an underlying pathology }\end{array}$ \\
\hline
\end{tabular}

value (ARV) and area under the curve (integrated EMG) in both absolute values (voltage) and relative to reference contractions including maximal, submaximal or reference voluntary contractions (MVC, SMVC, RVC respectively). ${ }^{34}$ Only studies which measure the level of activity of the muscle quantitatively will be included.

\section{Studies}

From scoping searches, it was determined that the highest level of evidence for this review is likely to comprise of observational studies.

The inclusion and exclusion criteria for this review are briefly summarised in table 1 .

\section{Information sources}

Information sources will be searched from inception to 21 August 2019. Specific search strategies using medical subject heading (MESH) terms have been developed to use where appropriate. The following databases will be used for searching: Web of Science, PubMed, MEDLINE (OVID Interface), EMBASE (OVID interface), ZETOC and CINAHL (EBSCO interface).

Hand searching of key journals will be conducted, including the Journal of Electromyography and Kinesiology, Clinical Neurophysiology, Muscle \& Nerve, Clinical Biomechanics and The Clinical Journal of Pain. Notable authors in the field will be contacted to identify relevant unpublished literature which is currently in preparation. Grey literature will be included in the search, and searches will be conducted using the British National bibliography for report literature, Opengrey and dissertation abstracts. Conference proceedings from 2017 to 2019 will be accessed, including the Congress of the International Society of Electrophysiology and Kinesiology (ISEK), the World Congress of Biomechanics (WCB), Society for Back Pain Research Annual Meeting (SBPR) and the World Congress on Low Back \& Pelvic Girdle Pain. To ensure completeness of the literature search, the reference lists of studies which are identified as eligible following the search will be hand searched to ensure that no relevant studies are missed.

\section{Search strategy}

The search will be conducted by the lead author (AS) and has been informed by subject-specific expertise and the completion of scoping searches. There will be no restrictions on the search in terms of the design, language, region or date. The keyword search strategy has been developed for MEDLINE (OVID interface) and incudes MESH keywords to ensure completeness of the search. The specific search terms will be modified to reflect differences in keywords and syntax between databases; however, the search strategy will remain consistent. A detailed search strategy for the Medline database has been included in online supplementary file 2 .

The lead author will search all information sources to identify relevant studies, and subsequently remove all duplicate studies. Then two authors (AS, AG) will independently screen the identified studies. Following the identification of all eligible studies, information extraction will be completed by AS, with accuracy checked by AG. The reviewers will not be blinded towards the authors, research group, or institutional information of the studies.

\section{Data management}

The results of the literature search, including the citation and abstract for each relevant study will be imported into EndNote X9 (Clarivate Analytics). Studies will be imported during the search, and duplicate studies will be identified and removed prior to the screening process. At this point, the remaining studies will be replicated into 
an individual folder for each reviewer for screening. In order to screen effectively, the full text for all potentially eligible studies will be retrieved and stored in EndNote X9. Screening will be accomplished using forms which have been developed to reflect the previously stated inclusion and exclusion criteria.

\section{Selection process}

The study selection process has been designed to reflect the best practice guidelines suggested by the Cochrane Back Review Group. ${ }^{25}$ Initial screening of search results will exclude studies in which it is clear from the title and abstract that the content is not relevant to objectives of this review. Where eligibility is unclear, the reviewers will read the full text of the article and use the screening tool to assess inclusion. The screening form will be used to objectively assess the study based on the inclusion and exclusion criteria. This form will identify studies as eligible, ineligible or unclear in terms of its inclusion in the review. In the event that a study is identified as unclear it will be discussed with the second reviewer to determine eligibility. If there is no consensus following this discussion, a third reviewer will be asked to determine the eligibility of the study. Furthermore, in the instance that one reviewer is an author on a potentially eligible study, screening for inclusion will be conducted by the remaining and third reviewer.

\section{Data collection process}

Data will be extracted by AS using a standardised form which has been created, based on the Cochrane data extraction template, using the objectives of the review and the inclusion criteria as a guide. ${ }^{26}$ The extracted data will be reviewed for accuracy by AG and any discrepancies discussed between reviewers, where resolution cannot be achieved, the third reviewer will determine which data are relevant. The standardised form will be piloted for completeness of data extraction on a subsection of studies and any necessary changes will be implemented prior to extraction from all eligible studies.

\section{Data items}

A summary of data items to be extracted from eligible studies is included in table 2 . Where data are missing from studies or the results are presented in an ambiguous way, the corresponding author will be contacted for clarification. If the clarification affects the eligibility of the study, and the author does not respond within a set timeframe, the study will be considered ineligible; however, it will be noted as excluded for ambiguity. If a study appears to use the same sample as another eligible study, the authors will be contacted to ensure that the results are not recorded in duplicate. In the instance that intramuscular EMG has been used in tandem with sEMG, the data from the intramuscular electrodes will be discarded, but the sEMG data will be preserved.

\section{Risk of bias}

Bias within individual studies will be assessed independently by each reviewer (AS, AG) with the
Table 2 Summary of items to be extracted from eligible studies using the standardised data extraction form

\begin{tabular}{ll}
\hline Information area & Data extracted \\
\hline Background & Authors \\
Year of Publication & Title \\
Methodology & Study Design \\
& Setting \\
& Sample Characteristics (Sample size, \\
& age, anthropomorphic data) \\
& LBP Characteristics (Duration, average \\
& pain, current pain, laterality) \\
& Task Information (Fatiguing, endurance, \\
& repeated, standing, length of recording, \\
& etc) \\
& Type of surface EMG (bipolar, linear \\
& array, HDEMG) \\
& EMG Processing (sampling frequency, \\
& filtering, offline processing) \\
& EMG Processing of signal amplitude \\
& (RMS, ARV, integrated EMG) \\
& Muscles measured \\
& Absolute or Normalised Activity values \\
& Normalisation Parameter and technique \\
& (MVC, SMVC, RVC) \\
& Amplitude Outcomes (Within subjects \\
& Pre/Post, between subjects pre/ \\
& post, changes throughout, systematic \\
differences between groups) & Effect Sizes where reported \\
\hline Results & \\
\hline &
\end{tabular}

Newcastle-Ottawa scale (NOS). ${ }^{35}$ The scale is designed for use within all types of observational studies and considers the selection and comparability of the sample as well as the assessment of the outcomes. ${ }^{36}$ Each study will be designated a star rating from 0 to a maximum of 9 stars, for which guidelines exist to convert to Agency for Healthcare Research and Quality standards. ${ }^{35} 37$ Using these guidelines, studies selected for inclusion into the review will be graded as 'Good', 'Fair' or 'Poor' quality with the star ratings for each subheading also reported.

Previous investigations into the reliability and the validity of NOS have presented mixed results, showing in some cases lower reliability when compared with other tools. ${ }^{3638}{ }^{39}$ However, it has previously been reported that for observational studies 86 individual risk of bias tools have been created, with no clear preferable candidate for determining risk of bias. ${ }^{40}$ For this review, it was decided to follow the advice of Hootman and colleagues, who determined that the NOS remains preferable to alternate risk of bias tools due to its moderate to high reliability, ease of use and face validity across observational designs. ${ }^{36}$

\section{Data synthesis}

In order to be included in meta-analysis, the outcomes and the methodology of the considered studies must be homogenous; as such, the possibilities for meta-analysis will become clear following data extraction. Thus, the 
remainder of this section speculates on the expected search results; however, changes may be required following data extraction.

The heterogeneity of the eligible studies will be assessed using the following characteristics:

- Type of sEMG used

- Measurement of muscle activity outcome (eg, is the measurement relative or absolute)

- Which muscles were considered

- Task completed (eg, fatiguing, endurance, repeated, standing)

Where homogeneity is sufficient between groups within these categories, then inclusion within meta-analysis either as a large group or as several subgroups will be determined by both reviewers. If the included studies are clearly homogenous in methodology, or are not clearly heterogeneous, then each reviewer will independently place studies into appropriate groups for analysis. This grouping will be determined by each reviewer independently, based on factors that they believe will allow the best and most accurate comparisons to be made. Thus, these subgroupings are likely to be based on the methodological factors mentioned previously, for example, task completed and/or muscles considered, as appropriate for the eligible studies. If appropriate, reviewers can include studies in more than one subgroup (eg, one group for muscles and one group for task), however in this instance care will be taken to ensure that these subgroups are not included in the same meta-analysis. Where concurrence exists between reviewers for these groupings, metaanalysis will occur. If the reviewers do not broadly agree on groupings then the potential for meta-synthesis will be instead discussed by the reviewers. Where disagreement occurs at any stage that cannot be resolved by the primary reviewers, the third reviewer will determine the possibility of meta-analysis or meta-synthesis.

In the instance that the reviewers determine a metaanalysis is appropriate, a statistical test of heterogeneity will be performed, providing an $\mathrm{I}^{2}$ value for the heterogeneity of the sample. ${ }^{41}$ The $\mathrm{I}^{2}$ value will be reported as a percentage and interpreted as suggested by the Cochrane Handbook for Systematic Reviews of Interventions. ${ }^{26}$ Significance in the measure of heterogeneity as calculated by the chi-squared test, will be set at $p<0.1$. As in previous reviews, the groupings of studies will be eligible for meta-analysis if an $\mathrm{I}^{2}$ value of $<50 \%$ (low heterogeneity) is calculated. ${ }^{42}$ Groups which exceed this value will be ineligible for meta-analysis and so will instead be considered for meta-synthesis.

Comparisons of changes in muscle activity between studies may be very difficult to assess due to differences in signal processing, tasks and experimental design. However, where meta-analysis is determined to be appropriate, results will be extracted from the relevant studies and compiled, with changes normalised and reported as percentage changes in all studies. Where inadequate data are provided within the publication to allow this analysis, the authors will be contacted to provide further data. This compiled data will then be used to create Odds Ratios (ORs) investigating the relationship between LBP and changes in muscle activity across studies.

ORs are traditionally used for binary outcomes whereby the odds of one outcome or another can be easily compared. ${ }^{43}$ Thus, if appropriate for the studies identified within the review, here, it is suggested instead to use the ORs to allow comparisons between different subgroupings of tasks and muscles. ORs will be calculated from the proportions of studies within each subgroup to show either an increase or a decrease in the level of activity. This analysis will allow for the estimation of effects across tasks and muscles, and will indicate if any specific tasks or muscles have outlying results. Within subgroups, percentage changes in activity will be correlated with secondary outcomes, including the level and duration of pain, if reported, to investigate the what effect these have on lumbar muscle activity.

If the data are not sufficiently homogenous, a narrative synthesis will instead be applied to the data set, with more binary elements of analysis also included. The results will be distilled to provide results in the format of an 'increase', 'decrease' or 'no change' in activity in the muscle activity.

\section{Confidence in cumulative evidence}

The risk of publication bias will be assessed by conducting a detailed search of unpublished studies, contacting notable authors in the field and the inclusion of grey literature in the search. Relevant conference proceedings from the previous 2 years will be accessed and authors of presented studies which were not brought forward to publication will be contacted.

The pooled data will be assessed using the Grading of Recommendations Assessment, Development and Evaluation (GRADE) approach to evaluate the overall quality of the literature. ${ }^{44}$ GRADE approach will be applied to the groupings identified earlier, and will be carried out according to the guidelines. Thus, observational studies will initially be given a 'Low' rating for the quality of evidence, and then the quality of evidence can be either upgraded or downgraded from this point. ${ }^{45}$ Studies will be upgraded for factors such as large effect sizes or doseresponse relationships between LBP severity and activity. Studies could also be downgraded for factors including publication bias, indirect relationships with results or inconsistencies between studies. ${ }^{44}{ }^{45}$ Following this process, a final quality level will be given to the evidence, and will be either 'High', 'Moderate', 'Low' or 'Very Low' which can be interpreted in line with the GRADE guidance. $^{25} 4445$

\section{Patient and public involvement}

The topic of this review was discussed at our established patient and public involvement meetings. Patients will not be involved in the analysis and data collection of the systematic review. 


\section{Ethics and dissemination of results}

No ethical approval is required for this review, as it will only involve the collation of previously published literature. This systematic review will collect and collate results from the numerous studies which have investigated changes in superficial lumbar muscle activity in people with CNSLBP. While heterogeneity is expected, it is expected that this review will succeed in identifying common adaptations which may be muscle and task specific. Consequently, the results of this review, and any differences in muscle activity between individuals with and without LBP, have the potential to influence future research into LBP including potential rehabilitation approaches. The results of this review will be submitted for publication in a peer-reviewed journal and presented at conferences.

Twitter Andy Sanderson @AndyCSanderson, Alison B Rushton @abrushton, Nicola R Heneghan @HeneghanNicola and Deborah Falla @Deb_Falla

Contributors All authors contributed to the focus of the systematic review topic. AS drafted the initial protocol, with guidance and feedback at all stages from DF, EMV, AG, NRH and ABR. All authors have revised and reviewed each draft of the protocol and have approved the final manuscript.

Funding The authors have not declared a specific grant for this research from any funding agency in the public, commercial or not-for-profit sectors.

Competing interests None declared.

Patient consent for publication Not required.

Provenance and peer review Not commissioned; externally peer reviewed.

Data availability statement There are no data in this work.

Open access This is an open access article distributed in accordance with the Creative Commons Attribution Non Commercial (CC BY-NC 4.0) license, which permits others to distribute, remix, adapt, build upon this work non-commercially, and license their derivative works on different terms, provided the original work is properly cited, appropriate credit is given, any changes made indicated, and the use is non-commercial. See: http://creativecommons.org/licenses/by-nc/4.0/.

\section{ORCID iDs}

Andy Sanderson http://orcid.org/0000-0002-7892-1067

Alison B Rushton http://orcid.org/0000-0001-8114-7669

Nicola R Heneghan http://orcid.org/0000-0001-7599-3674

Deborah Falla http://orcid.org/0000-0003-1689-6190

\section{REFERENCES}

1 Hoy $\mathrm{D}$, March L, Brooks $\mathrm{P}$, et al. The global burden of low back pain: estimates from the global burden of disease 2010 study. Ann Rheum Dis 2014;73:968-74.

2 Hong J, Reed C, Novick D, et al. Costs associated with treatment of chronic low back pain: an analysis of the UK general practice research database. Spine 2013;38:75-82.

3 Hartvigsen J, Hancock MJ, Kongsted A, et al. What low back pain is and why we need to pay attention. Lancet 2018;391:2356-67.

4 Henschke N, Maher CG, Refshauge KM, et al. Prognosis in patients with recent onset low back pain in Australian primary care: inception cohort study. BMJ 2008;337:a171.

5 Traeger AC, Moseley GL, Hübscher M, et al. Pain education to prevent chronic low back pain: a study protocol for a randomised controlled trial. BMJ Open 2014;4:e005505.

6 Chou R. Low back pain (chronic). BMJ Clin Evid 2010;2010. [Epub ahead of print: 08 Oct 2010].

7 Falla D, Hodges PW. Individualized exercise interventions for spinal pain. Exerc Sport Sci Rev 2017;45:105-15.

8 Geisser ME, Ranavaya M, Haig AJ, et al. A meta-analytic review of surface electromyography among persons with low back pain and normal, healthy controls. J Pain 2005;6:711-26.

9 Marshall PW, Murphy BA. Muscle activation changes after exercise rehabilitation for chronic low back pain. Arch Phys Med Rehabil 2008;89:1305-13.
10 Russo M, Deckers K, Eldabe S, et al. Muscle control and nonspecific chronic low back pain. Neuromodulation 2018;21:1-9.

11 Bogduk N. Clinical anatomy of the lumbar spine and sacrum. 4th ed. Edinburgh: Elsevier/Churchill Livingstone, 2005: 250 p.

12 Christophy M, Faruk Senan NA, Lotz JC, et al. A musculoskeletal model for the lumbar spine. Biomech Model Mechanobiol 2012;11:19-34

13 Daggfeldt K, Thorstensson A. The mechanics of backextensor torque production about the lumbar spine. J Biomech 2003;36:815-25.

14 Abboud J, Nougarou F, Pagé I, et al. Trunk motor variability in patients with non-specific chronic low back pain. Eur J Appl Physiol 2014;114:2645-54

15 Falla D, Gizzi L, Tschapek M, et al. Reduced task-induced variations in the distribution of activity across back muscle regions in individuals with low back pain. Pain 2014;155:944-53.

16 Tucker K, Falla D, Graven-Nielsen T, et al. Electromyographic mapping of the erector spinae muscle with varying load and during sustained contraction. J Electromyogr Kinesiol 2009;19:373-9.

17 Merletti R, Farina D. Surface electromyography: physiology, engineering, and applications. Hoboken, NJ: John Wiley \& Sons, 2016.

18 Jiang N, Falla D, d'Avella A, et al. Myoelectric control in neurorehabilitation. Crit Rev Biomed Eng 2010;38:381-91.

19 Correia JP, Oliveira R, Vaz JR, et al. Trunk muscle activation, fatigue and low back pain in tennis players. J Sci Med Sport 2016;19:311-6.

20 Jubany J, Danneels L, Angulo-Barroso R. The influence of fatigue and chronic low back pain on muscle recruitment patterns following an unexpected external perturbation. BMC Musculoskelet Disord 2017;18:161.

21 Suehiro T, Mizutani M, Ishida H, et al. Individuals with chronic low back pain demonstrate delayed onset of the back muscle activity during prone hip extension. J Electromyogr Kinesiol 2015;25:675-80.

22 Sanderson A, Martinez-Valdes E, Heneghan NR, et al. Variation in the spatial distribution of erector spinae activity during a lumbar endurance task in people with low back pain. $J$ Anat 2019;234:532-42.

23 Drost G, Stegeman DF, van Engelen BGM, et al. Clinical applications of high-density surface EMG: a systematic review. J Electromyogr Kinesiol 2006;16:586-602.

24 Ehsani F, Arab AM, Jaberzadeh S. The effect of surface instability on the differential activation of muscle activity in low back pain patients as compared to healthy individuals: a systematic review of the literature and meta-analysis. J Back Musculoskelet Rehabil 2017;30:649-62.

25 Furlan AD, Pennick V, Bombardier C, et al. 2009 updated method guidelines for systematic reviews in the Cochrane back review group. Spine 2009;34:1929-41.

26 Green S, Je H. Cochrane handbook for systematic reviews of interventions, 2011. http://handbook-5-1.cochrane.org/: Version

27 Moher D, Shamseer L, Clarke M, et al. Preferred reporting items for systematic review and meta-analysis protocols (PRISMA-P) 2015 statement. Syst Rev 2015;4:1.

28 Shamseer L, Moher D, Clarke M, et al. Preferred reporting items for systematic review and meta-analysis protocols (PRISMA-P) 2015: elaboration and explanation. BMJ 2015;350:g7647.

29 Smith V, Devane D, Begley CM, et al. Methodology in conducting a systematic review of systematic reviews of healthcare interventions. BMC Med Res Methodol 2011;11:15.

30 Dionne CE, Dunn KM, Croft PR, et al. A consensus approach toward the standardization of back pain definitions for use in prevalence studies. Spine 2008;33:95-103.

31 Barbero M, Merletti R, Rainoldi A. Atlas of muscle innervation zones. Springer-Verlag Mailand, 2012.

32 Heydari A, Nargol AVF, Jones APC, et al. EMG analysis of lumbar paraspinal muscles as a predictor of the risk of low-back pain. Eur Spine J 2010;19:1145-52.

33 Tassinary LG, Cacioppo JT, Vanman EJ, et al. The Skeletomotor system: surface electromyography. Handbook of Psychophysiology. New York, NY, US: Cambridge University Press, 2007: 267-300.

34 van Dieën JH, Selen LPJ, Cholewicki J. Trunk muscle activation in low-back pain patients, an analysis of the literature. J Electromyogr Kinesiol 2003;13:333-51.

35 Wells GA, Shea B, O'Connell D, et al. The Newcastle-Ottawa scale (NOS) for assessing the quality of nonrandomised studies in meta-analyses. Available: http://www.ohri.ca/programs/clinical_ epidemiology/oxford.asp [Accessed 11 Dec 2018].

36 Hootman JM, Driban JB, Sitler MR, et al. Reliability and validity of three quality rating instruments for systematic reviews of observational studies. Res Synth Methods 2011;2:110-8. 
37 Luchini C, Stubbs B, Solmi M, et al. Assessing the quality of studies in meta-analyses: advantages and limitations of the Newcastle Ottawa scale. World J Metaanal 2017;5:80-4.

38 Losilla J-M, Oliveras I, Marin-Garcia JA, et al. Three risk of bias tools lead to opposite conclusions in observational research synthesis. $J$ Clin Epidemiol 2018;101:61-72.

39 Hartling L, Milne A, Hamm MP, et al. Testing the Newcastle Ottawa scale showed low reliability between individual reviewers. J Clin Epidemiol 2013;66:982-93.

40 Sanderson S, Tatt ID, Higgins JPT. Tools for assessing quality and susceptibility to bias in observational studies in epidemiology: a systematic review and annotated bibliography. Int J Epidemiol 2007;36:666-76.
41 Higgins JPT, Thompson SG, Deeks JJ, et al. Measuring inconsistency in meta-analyses. $B M J$ 2003;327:557-60.

42 Heneghan NR, Smith R, Rushton A. Thoracic dysfunction in whiplash-associated disorders: a systematic review and metaanalysis protocol. Syst Rev 2016;5:26.

43 Egger M, Smith GD, Phillips AN. Meta-analysis: principles and procedures. BMJ 1997;315:1533-7.

44 Goldet G, Howick J. Understanding grade: an introduction. J Evid Based Med 2013;6:50-4.

45 Balshem H, Helfand M, Schünemann HJ, et al. Grade guidelines: 3. rating the quality of evidence. $J$ Clin Epidemiol 2011;64:401-6. 